\title{
Anaesthesia for the Elderly Patient
}

Salma Sophie

Department of Anaesthesia, Aga Khan University Hospital, Karachi.

\begin{abstract}
As life expectancy increases, the number of geriatric patients coming for surgery and anaesthesia will make up an increasing portion of our practice. Advancing age, co-morbidities, altered pharmacokinetics and dynamics increase the morbidity and mortality of these patients.

The importance of doing a thorough preoperative evaluation and identifying risk factors cannot be over emphasized in this frail and vulnerable group. Not all elderly patients have medical problems, stressing that physiological age is more important than chronological age. Current medical history and physical activity is a good indicator of how the surgical procedure will be tolerated. Addressed in this article is Post Operative Cognitive Dysfunction (POCD) which is being recognized as a significant issue facing the elderly patient. It is one of the problems specifically related to the geriatric age group.
\end{abstract}

No ideal anaesthetic technique has been described, but if a thorough understanding of changes that occur in physiology and pharmacology is there, an optimal anaesthetic technique can be individually designed.

\section{Anaesthesia for the elderly patient}

Aging is an irreversible and progressive physiological phenomena characterized by degenerative changes in the structure and functional reserve of organs and tissues. ${ }^{1}$ Advances and improvement in medical science have increased life expectancy thus, peri-operative surgery and anaesthesia in the elderly patient has become an extremely important issue.

Elderly patients (arbitrarily defined as being over 65 years of age) are vulnerable to the adverse effects of anaesthesia because of their reduced margin of safety. Morbidity and mortality increases with advancing age, with a steep increase after the age of 75 years. ${ }^{2}$ The frequency of complications related to anaesthesia is $0.5 \%$ in patients $>80$ years old. ${ }^{3}$ In a case series of 795 patients 90 years and older (at Mayo clinic) 9.4\% experienced serious morbidity within 48 hours, mortality rate being $1.6 \% .{ }^{4}$ Emergency procedures are associated with a higher mortality rate regardless of age group. Abdominal and thoracic procedures have been shown by multiple studies to have a higher complication rate.5,6

Life expectancy in USA (2006) was determined as 75.02 years in males and 80.82 years in females. ${ }^{7}$ In Pakistan (2006) the figures showed 62.04 years in males and 64.44 in females.
The mechanisms that control aging process and life span remain unknown. The most recent concept suggests that cellular aging is due to a progressive failure of a genetically determined capacity, to clear random damage to mitochondrial DNA by free radicals. ${ }^{1}$ Clinically, it presents as a loss of functional reserve of organ systems, which is the safety margin available to meet additional demands imposed by trauma, disease, healing and convalescence. ${ }^{2}$

\section{Physiology of aging}

Central Nervous System (Central): The target organ for all anaesthetic agents is the Central nervous system (CNS). Aging produces a decrease in neural density and loss of 30\% brain mass by the age of 80 years (mostly grey matter). Neuronal activity, blood flow metabolism, auto-regulation and cerebrovascular response to $\mathrm{CO}_{2}$ remain intact in the absence of disease. ${ }^{8}$ Cerebral blood flow is reduced by 10 $20 \%$, an appropriate response to decreased $\mathrm{CMRO}_{2}$ and decreased cerebral mass. There is a depletion of brain neurotransmitters (catecholamines, serotonin, acetylcholine). These are associated with mood, memory and motor function and may partly account for their loss. Transmitter destruction occurs at an accelerated rate, exaggerating the consequences of central neurotransmitter deficiency. ${ }^{9}$ Dopamine uptake sites, transporters and levels are reduced as also are cortical serotonergic, alpha 2, beta and GABA binding sites .

Central Nervous System (Peripheral): Signs of peripheral CNS aging are marked by a loss of motor sensory and autonomic fibers, decrease in afferent and efferent conduction velocities and progressive decline of signal processing rate within the brain stem and spinal cord. ${ }^{1}$ There is a reduction both in the efficiency of nerve to muscle coupling, and in the number of muscle cells innervated by an axon, leading to denervation and atrophy of muscle. Plasma epinephrine and norepinephrine levels are increased, but responsiveness of aging autonomic end organs is reduced.

Cardiac Aging: Integrated cardiac function changes less than cardiac and vascular anatomy. ${ }^{1}$ Cardiac output is reduced only in proportion to decreased skeletal muscle and lean tissue mass. Ventricular pump function appears to be largely a reflection of conditioning and aerobic demand rather than chronological age.

Aging impairs diastolic filling, chronotropic and inotropic responses of the heart. Therefore ability of the older patient to cope with stress is compromised. Increased metabolic demands may not be met when maximal cardiac output and oxygen delivery are limited. ${ }^{10}$ Heart rate is reduced, due 
to decreased sympathetic activity, fibrotic infiltration or degenerative changes in conductive system. Loss of large artery elasticity produces a progressive rise in systemic BP and widening of pulse pressure.. Arterial stiffening progresses more rapidly in females, proposing endocrine mechanisms to explain gender role in this discrepancy. Impedance of stroke volume ejection, produces concentric LVH. Reduced ventricular compliance makes the heart, volume sensitive and volume intolerant. ${ }^{11}$

Veins are also subjected to a progressive decrease in compliance. This decrease exaggerates hypotension resulting from blood loss, as well as from peripheral pooling of blood with general or regional anaesthesia. ${ }^{12}$

Cardiac output decreases at a rate of $1 \%$ per year. At the age of 80 years it is decreased by $50 \%$ as compared to a 30 year old. ${ }^{2}$ This is due to increased myocardial stiffness, interstitial fibrosis, progressive atherosclerosis and increased amyloid deposits in the myocardium. Elderly patients are at an increased risk of developing hypotension because of decreased baroreceptor sensitivity, decreased response to beta stimulation and decreased response of renin/aldosterone/angiotensin systems. ${ }^{13}$

Pulmonary Aging: A number of striking anatomic changes occur in the respiratory system with age. ${ }^{14}$

Emphysema-like increase in parenchymal compliance causes ventilation perfusion mismatch, increased physiological shunting and reduced efficiency of gas exchange. Ventilation perfusion mismatch causes arterial oxygen tension to fall linearly with age. However, $\mathrm{CO}_{2}$ excretion is minimally affected. There is marked suppression of hyperventilation, in response to imposed hypoxia or hypercapnia putting these patients at a higher risk for developing respiratory failure. Anaesthesia, supine position and use of narcotics worsens hypoxia by increasing ventilation perfusion mismatch in an age group that already has blunted ventilatory reflexes. Impaired cough and laryngeal reflexes, decreased immune response and mucociliary clearance increases the risk of aspiration and post operative pneumonia. ${ }^{14}$

Pulmonary function tests: Pulmonary reserve decreases with increasing age. Vital capacity decreases $25 \mathrm{ml}$ per year after age 20. FEV1 falls by 0.2 liters per decade after age twenty. $\mathrm{PaO} 2$ decreases $4 \mathrm{~mm} \mathrm{Hg}$ per decade after age 20. There is a progressive increase in closing volume which may contribute to post-operative atelectasis. Flow rates including FEV1 decrease by $20-30 \%, \mathrm{MBC}$ is reduced by at least $50 \%$ at 70 years. ${ }^{13}$

There are 4 hallmarks of the aging process $^{14}$ :

1. Decline in compliance of bony thorax

2. Loss of muscle mass with weakening of muscles of respiration and decreased mechanical advantage

3. Decrease in alveolar gas exchange surface

4. Decrease in central nervous system responsiveness

Dynamic lung compliance becomes frequency dependent. As breathing rate increases, lung expansion becomes less effective, particularly in some areas, increasing maldistribution of ventilation to perfusion. Decreased efficiency of vascular distensibility and recruitment leads to decreased HPV.

Ventilatory muscle fatigue is likely to occur early. Elderly patients develop ventilatory inadequacy earlier for any given ventilatory load. The subjective feature of ventilatory inadequacy is dyspnoea. 1,13

\section{Hepato-renal and metabolic aging}

Hepatic: Aging produces a marked reduction in liver size. Forty to fifty percent of young adult hepatic tissue mass, involutes by age of 80 years. Liver and splanchnic blood flow is reduced leading to delayed drug clearance. Hepatic synthesis of cholinesterase is deficient in elderly males. Production of albumin by the liver is decreased, resulting in decreased plasma protein binding of drugs. Loss of well perfused hepatic tissue plays an important role for drugs, which require biotransformation. Reduced functional CNS reserve, amplifies the effect of even low concentrations of these drugs. ${ }^{1,13}$

Renal: Glomerular filtration rate declines by $6-8 \%$ per decade (parallels reduction in cardiac output). As many as half of the glomeruli in young adults may be gone or rendered non-functional by 80 years. Pituitary response to dehydration is compromised. There is a reduced ability to concentrate urine after water deprivation, so the ability to excrete an acid load is reduced. Renal compromise is undetected by routine laboratory screening, with decreasing muscle mass serum creatinine is within normal limits. There is a prolonged half life of drugs, which are dependent on renal excretion for elimination. Decreased ability of the kidney to regulate salt balance, especially under stress makes fluid and electrolyte balance more labile. Reabsorption of glucose is decreased in elderly patients, glucosuria maybe misleading in diagnosis of diabetes mellitus. These alterations are unlikely to have any clinical significance unless challenged by super imposition of an acute illness or stress of surgery or infection. ${ }^{12,13}$

Endocrine: Decline in secretion of growth hormone and Insulin like growth factor-I (IGF-I) may increase sympathetic nerve activity. Decreased growth hormone is a cause of age related intracellular dehydration, atrophy of skeletal muscle and increase in lipid fraction of total body weight. Progressive decline in androgens may be a cause for change in body composition, leading to decreased muscle mass and total body water, and increase in adipose tissue. ${ }^{1}$

Pharmacology: Pharmacokinetic variables are altered because of decreased renal and hepatic function, decreased protein binding and altered volume of distribution. Alteration in pharmaco-dynamics is because of reduced numbers of receptors. Altered protein binding can be explained by decreased circulating levels of albumin, and qualitative changes in protein which reduces binding effectiveness. Coadministered drugs may interfere with the ability of anaesthetic agent to bind with available protein binding sites. Thus 
highly protein bound drugs when given to elderly patients will lead to higher free drug levels and an enhanced delivery of the drug to the brain. ${ }^{15}$ Decrease in blood volume $20-30 \%$ by age 75 leads to an initial dispersion of dose in a contracted volume producing a higher than expected initial plasma drug concentration. Increased body fat leads to sequestration of anaesthetic agents at these sites with a gradual, protracted elution.. Combined with reduced hepatic and renal elimination leads to a more gradual fall in plasma drug concentration. ${ }^{15}$

\section{Anaesthetic requirement}

Inhalation agents: Aging decreases the MAC linearly, beginning in young adulthood. ${ }^{15}$ Reduction in inhalation anaesthesia requirement is due to fundamental neurophysiological changes in the brain. Typically $66-75 \%$ anaesthetic concentration is required by an 80 year old as compared to a young adult. ${ }^{1}$ Rate of induction maybe slowed because of decreased ventilation, but the dose of inhalation agent should not be increased to hasten induction. There is a general perception that in elderly patients, inhalation agents cause a larger decrease in blood pressure, at a given concentration of volatile agent compared to younger patients. Many factors account for the perceived greater haemodynamic sensitivity, but age related impairment of reflex heart rate responses to blood pressure, decreased myocardial contractility by inhalation anaesthetics and volume contraction might enhance the decrease. ${ }^{16}$

\section{Intravenous anaesthetic agents}

Age related changes in requirement for IV anaesthetic agents such as thiopentone and etomidate maybe due to a higher than expected initial plasma concentration of these drugs during the early phase of redistribution. Dose reductions for propofol are required with concurrent administration of any other induction agent, narcotics or benzodiazepines as anaesthetic depth is synergistically increased. ${ }^{17}$ Age related decrease in clearance of propofol results in a decreased maintenance anaesthetic requirement with age. Patients more than 80 years old exhibit less mental impairment with propofol compared to other agents. Its hypotensive side effects will require slow administration of a reduced dose, titrated to effect rather than bolus of a pre-selected standardized dose. ${ }^{18}$ The dose of propofol should be $1.0-1.5 \mathrm{mg} / \mathrm{kg}$ without opiods, $0.5-1.0 \mathrm{mg} / \mathrm{kg}$ with opioids especially when midazolam or ketamine is given. Mask induction with sevoflurane causes less hypotension than intravenous propofol induction. ${ }^{2}$ Hypotensive response of propofol is offset by intubation response and surgical stimulation but not with insertion of LMA.

Narcotics: The dose of narcotics should be reduced because of decreased volume of distribution and protein binding. Onset of analgesia is often delayed because of delayed receptor equilibration. There is an increased incidence of side effects of narcotics like nausea, respiratory depression and hypotension.

\section{Neuromuscular blocking agents}

Neuromuscular blocking agents (NMBA) should be limited to short or intermediate acting in all elderly patients in whom extubation is planned. Long acting NMBA are associated with long PACU stay and increased post-operative pulmonary complications. ${ }^{19}$ Intermediate acting relaxants vecuronium and rocuronium both of which depend on end organ elimination, have prolonged duration of action. Atracurium and cis-atracurium which are eliminated primarily by Hoffmann elimination, and depend on kidney as secondary means of elimination, do not have a prolonged duration of action. Mivacurium action is prolonged due to a decrease in plasma acetyl cholinesterase that accompanies aging. The choice of NMBA and monitoring depth of blockade is important as recovery is delayed. ${ }^{20}$ The density of neuromuscular blockade increases, with advancing age, increased duration of surgery, and hypothermia.

Emergence: End tidal gas monitoring significantly underestimates brain concentration of inhaled agents during emergence. ${ }^{2}$ This hysteresis effect is more marked with the more soluble agents isoflurane and sevoflurane than desflurane. Failure to take this into account leads to prolonged emergenqce. Use of beta blockers towards end of surgery helps in giving haemodynamic stability when inhalation agents are being withdrawn. ${ }^{21}$

\section{Regional anaesthesia versus general anaesthe- sia}

Geriatric patients should be handled gently and anaesthetic regime maintained in as simple a fashion as possible. During certain procedures regional anaesthesia has the advantage of ${ }^{15}$ :

1. Reduced postoperative negative nitrogen balance

2. Amelioration of stress response to surgery

3. Decreased incidence of postoperative thrombo-embolic complications

4. Decreased blood loss

5. Decreased postoperative mental confusion

Despite theoretical advantages for regional anaesthesia, studies using morbidity and mortality as end points do not reflect this when comparing regional with general anaesthesia, but in studies designed to reflect typical clinical practice regional anaesthesia wins out. ${ }^{2}$ Spinal anaesthesia presumably (by virtue of the risk of hypotension) maybe associated with more cerebro vascular risk in elderly patients undergoing repair of hip fractures. ${ }^{22}$ Selection of anaesthesia has to be individualized and influenced not only by the condition but also by the anaesthesiologist's skill and expertise. ${ }^{15}$ 


\section{Pre-operative evaluation}

Pre-operative mental status should be carefully assessed to facilitate evaluation of post operative cognitive dysfunction (POCD). ${ }^{13}$ Pre-operative visit provides an ideal opportunity to review medications and eliminate those that can be discontinued. Cognitive and sensory impairment may make eliciting a history difficult. ${ }^{2}$

Physical findings are modified by age and concomitant disease. Systolic ejection murmurs at the base of the heart may represent aortic valve cusp sclerosis rather than obstruction. Endocarditis is an increased problem in the elderly due to high prevalence of degenerative valvular heart disease and possibility of bacteremia associated with oral, bowel, bladder, biliary and pulmonary procedures. ${ }^{13}$

Intravascular volume status is critical because of the greater dependence of cardiac output on preload. Patients in congestive heart failure should not be over treated with diuretics to the point of volume depletion. Invasive monitoring should be considered in patients with significant cardiopulmonary problems or uncertain volume status.

Pre-operative Testing: Functional capacity provides an excellent estimate of reserve. It is defined as behaviours necessary to maintain activities of daily living. ${ }^{3}$ The disproportionate increase in perioperative risk to preexisting disease demonstrates the importance of pre anaesthesia screening. ${ }^{13}$ The anaesthetic assessment includes a history, physical exam and review of medical chart.

For low risk surgery the perioperative complication risk is $3 \% .^{2}$ For intermediate risk surgery, pre-op testing depends on the functional state of the patient. No invasive testing is required for patients with moderate functional capacity (4-7 mets) and intermediate clinical predictors (mild stable angina, prior myocardial infarction, compensated/prior congestive heart failure, diabetes mellitus) or for patients with poor functional capacity (1-4 mets) with minor clinical predictors (advanced age, abnormal EKG, non sinus rhythm, history of stroke, poorly controlled blood pressure). ${ }^{2}$

Pre-operative Laboratory Testing: Routine pre-op testing in geriatric surgical patients without any clinical indications, yields a small percentage of abnormal results. Appropriate testing should focus on co-morbidities and type of surgery. Selective laboratory testing as indicated by history and physical examination, (which will determine co-morbidities and surgical risk) seems to be indicated. ${ }^{23}$

Risk Assessment: General risk is quantified using ASA classification. Most of the elderly patients fall in ASA class II or III where overall mortality is low.This classification is prone to inter-observer bias. ${ }^{13}$

Prediction of Cardiac Morbidities: Cardiac complications can be predicted using Goldmans cardiac risk index which takes age as an independent risk factor. Though this index has predictive value the sensitivity is low as reflected by the high prevalence of coronary artery disease unmasked by stress of surgery. A study by Pedersen et al ${ }^{24}$ reports a $16.7 \%$ cardio- vascular complication rate in 80 year old compared to $2.6 \%$ in those less than 50 years. In the absence of specialized tests for estimating preoperative heart function, the goal should be to optimize symptomatic complaints as much as possible before surgery. ${ }^{5}$ A variety of diagnostic studies (thallium/dobutamine stress testing, prolonged ambulatory holter monitoring) are available for risk stratification but the value of interventions, made on the basis of abnormalities of such tests, has not been established. Invasive monitoring has been recommended to identify abnormal haemodynamics in elderly patients undergoing high risk procedures, but it remains unclear whether the benefits are justified by the attendant risk. ${ }^{13}$

Beta-Adrenergic Blockade: Beta blockers are recommended for patients who are not receiving them to reduce risk of perioperative MI (3 or higher cardiac risk index criteria) or intermediate risk patients (1-2 cardiac risk index criteria and any 2 minor criteria). ${ }^{2}$ Minor critical criteria include age 65 years or greater, hypertension, current smoker, elevated serum cholesterol and type 2 diabetes mellitus. Cardiac risk index consist of high risk surgery (intraperitoneal, thoracic, supra-inguinal vascular) ischaemic heart disease, cerebrovascular disease, type 1 diabetes mellitus and chronic renal failure (serum creatinine at least $2.0 \mathrm{mg} / \mathrm{dl}$ ). Target heart rate is 55-65 b.p.m. and systolic blood pressure $>100 \mathrm{~mm} \mathrm{Hg}$. Data suggests that withholding of beta blockers in patients taking them chronically, increases the risk of perioperative ischaemia. Most peri-operative myocardial infarctions occur on the day of surgery. ${ }^{2}$ Evidence is accumulating that diastolic blood pressure must be maintained within $10 \%$ of baseline and certainly above $60 \mathrm{~mm} \mathrm{Hg}$ in elderly patients.

Prediction of Pulmonary Complications: Preoperative optimization of respiratory function is important. Prior history of congestive heart failure and neurological problems increases the odds of adverse pulmonary events. Pulmonary complication rate has been quoted as $10.2 \%$ over 80 year age. ${ }^{24}$

Smoking, low serum albumin levels, prior respiratory disease, presence of obstructive pulmonary disease and old age increase the frequency of pulmonary complications. These include pneumonia, atelectasis, hypoxaemia and need for prolonged ventilation. Pulmonary function guidelines that correlate with increased pulmonary morbidity and mortality are: $\mathrm{MBC}<50 \%$ predicted, an $\mathrm{FEV} 1<2$ litres and $\mathrm{PaCO} 2>45$ $\mathrm{mm} \mathrm{Hg}$. Precise quantification of risk by degree of abnormality is not possible. 13,14

\section{Special concerns}

Pre-Oxygenation: Maximum pre-oxygenation is required because elderly patients desaturate faster and the time to peak relaxation from succinylcholine is also increased. Maximum pre-oxygenation is attained when along with arteriolar and alveolar compartments, venous and tissue compartments are also filled with oxygen. ${ }^{2}$ Maximum oxygen in a short period of time is 8 deep breaths of $100 \%$ oxygen in 60 seconds with 
an oxygen flow of 10 liters/minute. Elderly patients are more likely to suffer a cardiac event from desaturation. 25,26

Hypothermia: Elderly patients are not immune to the adverse effects of hypothermia which are bleeding, decreased immune function and decreased wound strength. ${ }^{27}$

Hypothermia is more pronounced and lasts longer, due to intrinsic factors (low BMR, hypothyroidism, high ratio of surface area to body mass). ${ }^{13}$ Impairment of autonomic mechanisms for thermoregulation, cold induced vasoconstriction and shivering in the elderly is delayed intraoperatively, until core temperature falls, to lower than, that in young adults. Younger patients shiver at $36.1^{\circ} \mathrm{C}$ while patients more than 80 years will not shiver till core body temperature decreases to $35.2^{\circ} \mathrm{C}$. Oxygen consumption is increased $38 \%$ over non shivering levels. Reduction in skeletal muscle mass, decreases post-op shivering thus limiting the rate at which temperature homeostasis can be re-established. Inhibition of thermoregulatory responses by anaesthetics is greatly exaggerated in elderly patients, prolonging clearance of anaesthetics which further renders them more susceptible to post operative hypothermia. ${ }^{26}$ Rewarming may precipitate sudden hypotension as a result of vasodilation and hypovolaemia. $^{13}$

\section{Post-operative Cognitive Dysfunction (POCD)}

Aging CNS with reduced functional reserve makes the elderly patient more susceptible to POCD. Acute disturbance in consciousness and cognitive function is twice as common in the elderly. The symptoms for POCD may be related to, further decreases in already low levels of neurotransmitters. Under anaesthesia etiological factors are hypotension, hypoxia, drug interaction, depression, dementia, alcohol abuse and metabolic disturbances. Anaesthetics which can produce delirium are ketamine, benzodiazepines and propofol, anticholinergics, atropine and scopolamine.

Pre-operative neurological disease increases rate of POCD. Data suggests anaesthesia itself may be a factor. Risk factors are increasing age, duration of anaesthesia, second surgery, post operative infection, preoperative neurologic disease and respiratory compromise. No particular anaesthesia technique is implicated.

The fact is we still do not know what anaesthetic drugs or techniques do to the elderly brain, and in what way their actions are different from their actions on the younger brain. ${ }^{28}$ Until more definitive studies are available, it is better to avoid hypoxia, hypercarbia and provide adequate post operative pain relief. $5,9,13,15$ It has been observed that POCD is less frequently seen when elderly patients undergo outpatient surgery. In this setting they receive fewer medications and are allowed to return sooner, to normal surroundings. ${ }^{15}$

Post operative Pain: Inadequately controlled postoperative pain can lead to slow recovery and POCD. ${ }^{15}$ The physicians are hesitant to give a "good" dose of pain medication because of the side effects of narcotics. Dementia, aphasia and cognitive impairment renders it very difficult for the physician to assess the level of pain. For geriatric patients, the oral route is simple and cost effective as intramuscular injections are painful, and absorption is unpredictable due to less muscle and more fat. Parenteral NSAIDs/Opiods with short half lives can be prescribed for mild to moderate pain, being careful of gastro-intestinal, renal and platelet effects. Meperidine must be used with caution as its metabolites rely on renal elimination. It is best to start with $25-50 \%$ of usual adult dose. Patients should be monitored for sedation and respiratory depression. ${ }^{29}$

Nutrition: Protein calorie malnutrition is seen in $20 \%$ of elderly patients. Albumin, a marker of nutritional status, is also a surrogate marker of preoperative health status. Serum albumin inversely correlates with post-op complications and mortality rates. ${ }^{5}$ Use of enteral or parenteral hyperalimentation must be balanced, considering patient complications. ${ }^{13}$

Ethical Concerns: The physician should respect the patient's autonomy and obtain consent, free of undue influence or coercion. Consent must be taken after sufficient information is given to the decision makers, who, should be able to understand the information given to them. The patients have the right to refuse medical therapy, even if it is in disagreement with the physician. Determining that an elderly patient has the capacity to make a medical decision can be a challenge for the anesthesiologist. When patients are too impaired a surrogate decision maker has to be involved.

A bias against performing surgery in the elderly still exists, due to misconceptions of age as a surgical risk. The extent of bias is difficult to quantify. If surgery can improve the quality of the remaining years, there is little ethical or moral basis for denying appropriate therapy based on age alone. Early surgical treatment should be considered whenever possible because delaying surgery, because of the patient's age is not supported by literature. $5,13,30$

\section{Conclusion}

The elderly often require more extensive preoperative and postoperative care than younger patients undergoing equivalent procedures. These patients are more likely to require intensive care. Aging alone does not significantly influence operative risk. Chronic diseases associated with advanced age increases that risk. Perioperative management should focus on diagnosis and optimization of these comorbidities and meticulous perioperative hemodynamic control. The degree of preparedness and acumen of the anesthesiologist is more important than the anaesthesia technique and agents.

The adage "start low, go slow" still holds true, but more research and education in the geriatric age group will improve outcome and help us to develop expertise in this relatively "young" subspeciality of geriatric anaesthesia. 
Recent data suggests most of the surgery is done, to improve quality of life rather than to increase survival. It seems reasonable to let patient preferences and their perception of symptoms assume dominant roles in decision making. Although different individuals age in different ways and degrees, clearly they all require greater vigilance and more active intervention to guide them safely through surgery and anaesthesia.

\section{References}

1. Muravchik S. Pharmacological changes of aging. 53rd ASA Annual Meeting Refresher Course Lectures \#19. 2002; pp 1-7.

2. Raymond R. Anesthetic management of the elderly patient. 53rd ASA Annual Meeting Refresher Course Lectures \#321. 2002; pp 1-7.

3. Warner D, Warner M. Anesthetic risk and the elderly. Syllabus on Geriatric Anesthesiology, ASA. 2002;pp 1-4.

4. Warner $\mathrm{H}$, Lobdell $\mathrm{CM}$ et al. Outcomes of surgery in patients 90 years of age and older. JAMA 1989; 261:1909; pp 1-4.

5. Liu L, Leung J M. Peri-operative complications in elderly patients. Syllabus on Geriatric Anesthesiology. 2002; pp 1-4.

6. Barnett S R. Pre-anesthetic evaluation for the elderly patient. Syllabus on Geriatric Anesthesiology, ASA. 2002; pp 1-3.

7. CIA: The World fact book [online] updated Feb 2007. www.cia.gov/cia/publications/factbooks/docs/profilguide.html

8. Thereault J. Aging and the central nervous system. Syllabus on Geriatric Anesthesiology ASA. 2002; pp 1-3/

9. Crosby G. Aging anaesthesia and brain. Implications for your and your patients.53rd ASA Annual Meeting Refresher Course Lectures \# 162. 2002; pp 17.

10. Amar D, Zhang H, Leung DHY, Roistacher N, Kadish AH. Older age is the strongest predictor of postoperative fibrillation. Anesthesiology 2002; 96:3526.

11. Tasch M D. Cardiovascular and autonomic nervous system aging. Syllabus on Geriatric Anesthesiology ASA 2002; pp 1-3

12. Barlow I. Peri-operative renal insufficiency and failure in elderly patients Syllabus on Geriatric Anesthesiology ASA 2002; pp 1-3.

13. Francis J Jr. Surgery in the elderly. David R Goldman, Frank H Brown, David M Guarneri. Peri-operative medicine 2nd ed. USA: McGraw-Hill, Inc. 1994; pp 385-94.

14. Ross B K. Aging and the respiratory system. Syllabus on Geriatric Anesthesiology ASA. 2002; pp 1-5.

15. McLeskey $\mathrm{C}$ H. Pharmacokinetic and pharmacodynamic difference in the eld erly. Syllabus on Geriatric Anesthesiology ASA 2002; pp 1-7.

16. Ebert T J. Physiology of the cardiovascular effects of general anaesthesia in the elderly. Syllabus on Geriatric Anesthesiology, ASA. 2002; pp 1-3.

17. Andreasen A I, Knopes K D. Induction agents. Syllabus on Geriatric Anesthesiology, ASA. 2002; pp 1-3.

18. Andreasen A I, Knopes K D. Opioids in the elderly. Syllabus on Geriatric Anesthesiology, ASA. 2002; pp 1-3.

19. Ballantyne J C, Y Chang. The impact of choice of muscle relaxant on postoperative recovery time: a retrospective study. Anaesth Anal 1997; 85:476-82.

20. Lien CA. Muscle relaxant selection and administration. Syllabus on Geriatric Anesthesiology, ASA. 2002; pp 1-3.

21. Zaugg M, Tagliente T, Lucchinetti E, Jacobs E, Krol M, et al. Beneficial effects from beta- adrenergic blockade in elderly patients undergoing non-cardiac surgery. Anesthesiology 1999; 91:1674.

22. Urwin S C, Parker M J, Griffiths R. General versus regional anaesthesia for hip fracture surgery: a meta analysis of randomized controlled trials. Br J Anaesth 2000; 84:450-5.

23. Dzankic S, Pastor D, Gonzalez c, Leung J M. The prevalence and predictive value of abnormal preoperative laboratory tests in elderly surgical patients Anesth Analg 2001;93:301-8.
24. Pedersen T, Eliasen K, Henriksen E. A prospective study of risk factors and cardiopulmonary complications associated with anaesthesia and surgery .Risk indicators of cardiopulmonary morbidity. Acta Anaesthesiol Scand 1990; 34:14455 .

25. Baraka A S, Taha S K, Aouad M T, El-Khatib M F, Kawkabani N I. Pre-oxygenation: comparison of maximal breathing and tidal volume breathing techniques. Anesthesiology 1999; 91:612.

26. Baraka A S, Taha S K, Kawkabani N. Editorial views. Pre-oxygenation. Best method for both efficacy and efficiency? Anaesthesiology 1999; 91:603-5.

27. Lien C A. Thermoregulation in the elderly. Syllabus on Geriatric Anesthesiology. ASA 2002.

28. Severn AM. Time to light the grey touch paper Editorial 11. Br J Anaesth 2001;87: 533-6.

29. Chin M L. Postoperative pain control in the elderly patient. Syllabus on Geriatric Anesthesiology, ASA. 2002; pp 1-6.

30. Norman G A V. Ethical challenges in the anesthetic care of the geriatric patient. Syllabus on Geriatric Anesthesiology, ASA. 2002; pp 1-5.. 